\title{
Effect of Substrate Orientation on Melt Pool during Multi-Layer Deposition in V-Groove with Gas Metal Arc
}

\author{
Pradip Aryal $^{1}$, Kjell Hurtig¹, Fredrik Sikström ${ }^{1}$, Håkan Nilsson², Isabelle Choquet ${ }^{1}$ \\ ${ }^{1}$ University West \\ Department of Engineering Science, 46186 Trollhättan, Sweden \\ pradip.aryal@hv.se; kjell.hurtig@hv.se; fredrik.sikstrom@hv.se; isabelle.choquet@hv.se \\ ${ }^{2}$ Chalmers University of Technology \\ Mechanics and Maritime Sciences, Fluid Dynamics, SE-412 96 Gothenburg, Sweden \\ hakan.nilsson@chalmers.se
}

\begin{abstract}
Thermo-fluid dynamic and experimental approaches are used to investigate the influence of $20^{\circ}$ uphill, downhill and sideway substrate orientation during metal deposition over a previously deposited bead in a V-groove. The computational fluid dynamic model with free surface deformation and metal transfer gives insight into the melt pool flow and causes of defect formation observed on the solidified beads. The experimental metallographs, high-speed images and computational results show good agreement. It is found that the deposition of a second layer on a smooth first layer cooled down to room temperature leads to large changes in melt pool flow pattern at $20^{\circ}$ substrate inclination compared to flat condition. It results in undercut and humps with the uphill orientation and undercut with the side inclination. Therefore, lower angle range is necessary for multilayer gas metal arc deposition for these two last configurations.
\end{abstract}

Keywords: metal deposition, gas metal arc welding, V-groove, substrate orientation, melt flow, reinforced bead, hump, OpenFOAM

\section{Introduction}

Metal deposition for multi-pass joining and additive manufacturing with gas metal arc (GMA) processing using nonhorizontal substrate orientation would avoid workpiece repositioning necessitating manipulation by large and accurate robots when manufacturing large components. However, the freedom for tilting the arc axis to maintain it normal to a non-horizontal substrate can be limited by defect formation such as overflow, undercut and hump. The effect of the welding position on the molten pool behaviour and resulting bead geometry was studied both experimentally (e.g., Park et al. [1]) and numerically (e.g., Hu et al. [2], Cho et al. [3]) with GMA processing, single bead deposit, and flat, overhead, and vertical welding position. These authors observed that while the flat position generated continuous bead without noticeable defects, the overhead and vertical up positions were prompt to hump formation. Vertical down position increased bead width, while it reduced height and penetration depth. Besides, it is known that for multi-layer deposition the melt pool can have remarkably different behaviour in the root pass and in the subsequent layers for which it becomes more sensitive to the process parameters. However, the effect of non-flat welding position in GMA multi-layer deposition is to our knowledge poorly documented. This study thus aims at understanding this effect on melt pool thermal flow, free surface deformation, bead geometry, possible defect formation when depositing above the root pass. It is known from earlier studies that overhead and vertical position cannot be an option with GMA heat source. Therefore, in this study the welding position is varied by only $20^{\circ}$ uphill, downhill, and sideway to investigate, using both experimental and computational fluid dynamics approaches, whether these conditions could provide an acceptable process window.

\section{Experimental setup}

Figure 1(left) shows an overview of the experimental setup. A precise movement ABB robot operated a gas metal arc torch with TPS-4000 Fronius power supply. It was used to deposit Invar 36 at the travel speed of $7.5 \mathrm{~mm} / \mathrm{s}$ from a $1.2 \mathrm{~mm}$ diameter electrode wire on Invar 36 plates with same nominal composition. The deposition was shielded by a mixture of $98 \% \mathrm{Ar}$ and $2 \% \mathrm{CO}_{2}$ flowing at the rate of $15 \mathrm{l} / \mathrm{min}$. The plates $(200 \mathrm{~mm} \times 143.5 \mathrm{~mm} \times 10.6 \mathrm{~mm})$ were prepared with a Vgroove at $60^{\circ}$ with no root gap and mounted on the worktable. This table that can be rotated about its central horizontal axis 
was inclined to position the substrate as illustrated in Fig. 1(right): flat (reference angular position at $0^{\circ}$ ), downhill (at $-20^{\circ}$ ), uphill $\left(\right.$ at $20^{\circ}$ ), and side inclined (at $20^{\circ}$ ). A data acquisition system was integrated with this setup to measure the arc current and voltage at a sampling frequency of $4 \mathrm{kHz}$. A vision camera was mounted on the robotic arm and focused behind the nozzle region to capture melt pool images at a frequency of $100 \mathrm{~Hz}$. For each substrate positioning two layers of metal were deposited using the GMA welding system. The torch axis was in each case perpendicular to the substrate, the arc length was approximately $5 \mathrm{~mm}$, and uniform contact-tip-to-work distance was maintained. The first layer deposit (root pass) generated continuous and uniform bead without noticeable defects. It was let to cool down to room temperature and the reinforced bead was 3D scanned before the second layer was deposited. A fifth-degree polynomial that curve fit the profile of the root pass bead reinforcement along the transverse direction was generated.

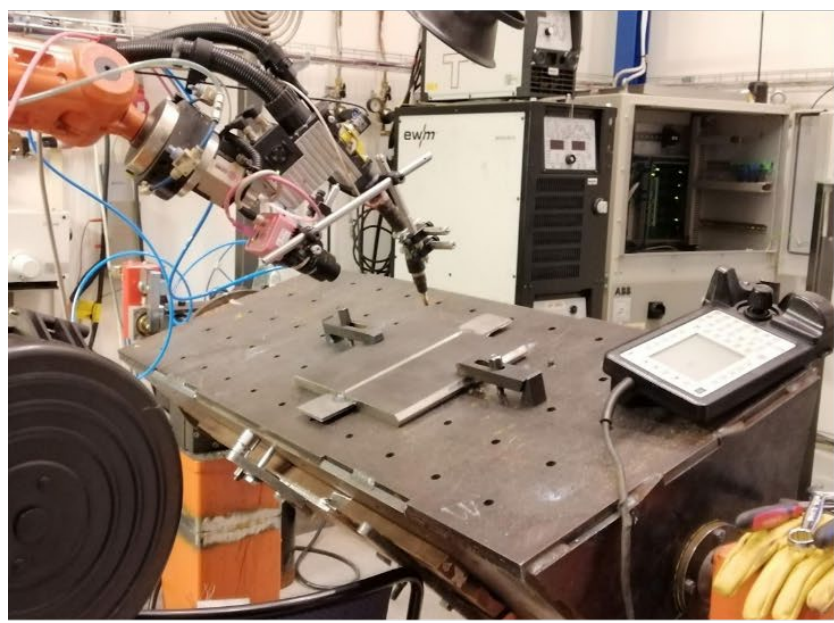

a)

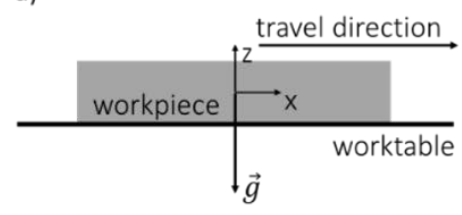

c)

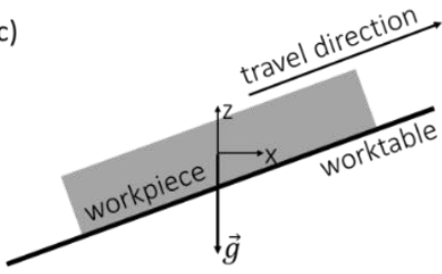

b)

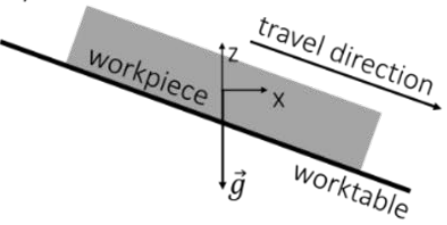

d)

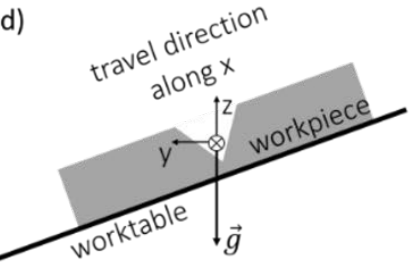

Fig. 1 : Left - Experimental setup; right - schematic of the substrate orientation: a) flat, b) downhill, c) uphill, d) side inclined.

\section{Mathematical model}

A transient 3-dimensional melt pool model with metal alloy melting, re-solidification, metal transfer, and free surface deformation was implemented in the open-source software OpenFOAM ${ }^{\circledR}$. It assumes incompressible and Newtonian fluids (atmosphere and liquid alloy with the Boussinesq approximation) and laminar flow. The alloy-gas interface is tracked with a volume of fluid method and the solid-liquid alloy transition region with a mushy zone approach. The model consists in the following system of equations governing mass, momentum, thermal energy, and metal volume fraction, respectively:

$$
\begin{gathered}
\partial_{t} \rho+\nabla \cdot(\rho \overrightarrow{\mathrm{u}})=\dot{\rho}_{\text {drop }} \\
\partial_{t}(\rho \overrightarrow{\mathrm{u}})+\nabla \cdot(\rho \overrightarrow{\mathrm{u}} \otimes \overrightarrow{\mathrm{u}})=-\nabla p+\nabla \cdot\left[\mu\left(\nabla \overrightarrow{\mathrm{u}}+(\nabla \vec{u})^{T}\right)-\frac{2}{3} \mu(\nabla \cdot \vec{u}) I\right]+\rho_{m} \vec{g}\left[1-\beta\left(T-T_{m}\right)\right] \\
-C_{D} \frac{\left(1-f_{l}\right)^{2}}{f_{l}^{3}+\varepsilon_{D}} \vec{u}+\left[\sigma \kappa \vec{n}+\frac{d \sigma}{d T}(\nabla T-\vec{n}(\vec{n} \cdot \nabla T))+p_{\mathrm{arc}}\right]|\nabla \alpha| \frac{2 \rho}{\rho_{l}+\rho_{g}}+\vec{F}_{\mathrm{arc}} \\
\partial_{t}\left(\rho c_{p} T\right)+\nabla \cdot\left(\rho c_{p} T \vec{u}\right)=\nabla \cdot(k \nabla T)+h_{s f}\left[\partial_{t}\left(\rho f_{l}\right)+\nabla \cdot\left(\rho \vec{u} f_{l}\right)\right]+\dot{Q}_{\mathrm{arc}} \\
\partial_{t} \alpha+\nabla \cdot(\alpha \overrightarrow{\mathrm{u}})+C_{\alpha} \nabla \cdot\left[\alpha(1-\alpha) \vec{u}_{c}\right]=\dot{\alpha}_{\text {drop }}
\end{gathered}
$$

where $t$ is the time. The variable $\vec{u}$ is the velocity vector, $p$ the pressure, $T$ the temperature and $\propto$ is the volume fraction of metal. The one-fluid density is the volume-weighted average $\rho=\alpha \rho_{m}+(1-\alpha) \rho_{g}$, where $\rho_{m}$ and $\rho_{g}$ are the metal and the 
atmospheric gas density, respectively. The source terms of metal mass, $\dot{\rho}_{\mathrm{drop}}$, and volume fraction, $\dot{\alpha}_{\mathrm{drop}}$, related to metal transfer in the form of molten droplets are specified hereafter. $I$ denotes the identity matrix. The one-fluid viscosity $\mu$, specific heat capacity $c_{p}$, and thermal conductivity $k$ are volume-weight averaged using the temperature dependent properties of Invar36 alloy [4]. In the buoyancy force $\vec{g}$ is the gravitational acceleration, $\beta$ the coefficient of volume expansion for the liquid alloy [4], and $\rho_{m}$ the alloy density at the melting temperature $T_{m}$. In the second line of Eq. (2) the first term is the Darcy damping. It is active in the mushy zone where the fraction of liquid alloy, $f_{l}$, is strictly bounded between zero and one. This fraction is a function of temperature, as defined in [5]. The Darcy constants are set to $C_{D}=10^{7}$ and $\varepsilon_{D}=10^{-3}$. The surface forces are estimated using the sharp surface force model introduced by Shams et al. [6]. As the densities of the two fluid phases differ by several orders of magnitude, the multiplier $2 \rho /\left(\rho_{m}+\rho_{g}\right)$ introduced by Brackbill et al. is applied [7]. The capillary and thermocapillary forces depend on the surface tension, $\sigma$, the unit vector normal to the free surface, $\vec{n}$, and the surface curvature, $\kappa$. The latent heat of fusion is denoted $h_{s f}$. In equation (4), the third term is the free-surface sharpening term, which is a function of the numerical compression velocity, $\vec{u}_{c}$, [8]. The numerical compression factor is set to $C_{\alpha}=1$ to satisfy conservation. The effect of the arc is modeled through the arc pressure, $p_{\text {arc }}$, the electromagnetic force $\vec{F}_{\text {arc }}$, and the heat source, $\dot{Q}_{\text {arc }}$. These three source terms are respectively estimated using the expression proposed by Tsai and Eagar [9], by Kou and Sun [10], and

$$
\dot{Q}_{\mathrm{arc}}=\frac{\eta_{\mathrm{arc}} V I}{2 \pi \sigma_{q}^{2}} \exp \left(-\frac{r^{2}}{2{\sigma_{q}}^{2}}\right)
$$

where $r$ is the radial distance from the center of the arc, $V$ the arc voltage and $I$ the arc current. The arc efficiency, $\eta_{\text {arc }}$, is estimated from the total GMA process efficiency $\eta=0.8$ [11], and the droplet efficiency according to [12]. The same value of the distribution parameter $\sigma_{q}$ is assumed for the heat flux, the arc pressure, and the current density according to the relationship proposed in [13]. Based on the experimental measurements, $V=25.2 \mathrm{~V}, I=270 \mathrm{~A}$ and $\sigma_{q}=1.4 \mathrm{~mm}$.

Figure 2 shows the computational domain of dimensions $60 \mathrm{~mm} \times 20 \mathrm{~mm} \times 13 \mathrm{~mm}$, which is shorter and narrower than the experimental substrate. For the uphill configuration, a thicker atmosphere layer of up to $\mathrm{z}=23 \mathrm{~mm}$ was used to capture the alloy deposition (see section 4). The results presented below were computed with a mesh size of $0.2 \mathrm{~mm}$ uniform in $\mathrm{x}, \mathrm{z}$, and in $-6 \leq \mathrm{y} \leq 6 \mathrm{~mm}$. A cell-to-cell expansion ratio of 1.2 was applied outside this region. The root pass bead profile scanned during the experiment served to initialise the first layer in the $\mathrm{V}$-groove, as shown in Fig. 2. For the configurations symmetric about $\mathrm{y}=0$, only one half of the domain was simulated; this concerns the flat, uphill, and downhill positioning.

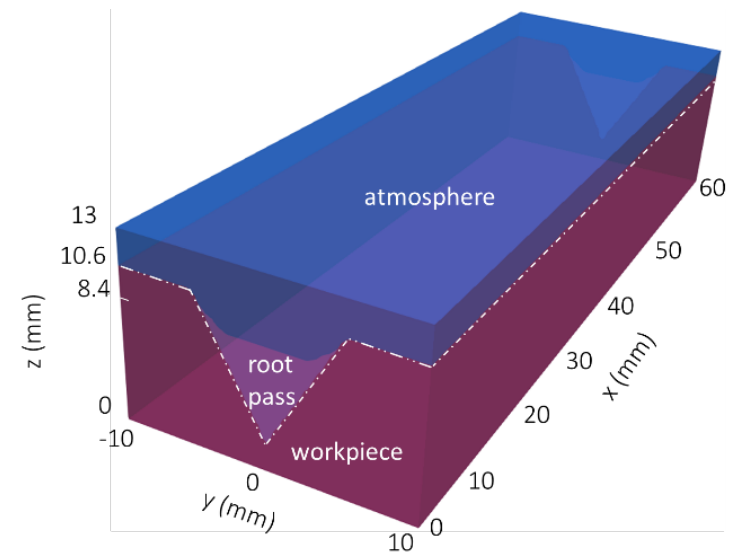

Fig. 2: Computational domain after initializing the root pass in the V-groove. 
The test cases were computed with an arc torch initially in $\mathrm{x}=10 \mathrm{~mm}$, so that the alloy remained solid on the domain boundaries. The boundary condition for the gas phase was zero velocity gradient if the flow was outward. Otherwise, the computed pressure was used to determine the magnitude of the normal velocity at the boundary face. The metal alloy had zero-velocity and continuous temperature gradient conditions at the boundaries, making the workpiece semi-infinite for heat

conduction. Based on experimental measurements the source terms $\dot{\rho}_{\text {drop }}$ and $\dot{\alpha}_{\text {drop }}$ reproduced spherical droplets of radius $0.54 \mathrm{~mm}$ transferred from the electrode wire at the periodic frequency of $207 \mathrm{~s}^{-1}$. The location of the arc and droplet injection was moved at the welding travel speed for a period of $6 \mathrm{~s}$. An adjustable time stepping with maximum CFL number of 0.1, and maximum time step of $10^{-5} \mathrm{~s}$ was applied. The pressure-velocity coupling was computed with a PISO algorithm. The convergence criteria imposed on the (final) residuals at each physical time step when solving for $\alpha, p, \vec{u}$ components and $T$ was $10^{-12}, 10^{-10}, 10^{-8}$, and $10^{-8}$, respectively.

\section{Results and discussion}

Figure 3 presents computed results showing the melt pool followed by the resolidified bead and experimental postprocess bead for the $20^{\circ}$ uphill case (different scales). A non-continuous bead with intermittent humps can be seen in the right. The computational results also show an unstable melt pool with humping bead geometry. As the computational domain was shorter than the experimental substrate, the simulation results did only predict the formation of the first hump. With the other configurations, experimental and computed process and bead surface were all smooth.
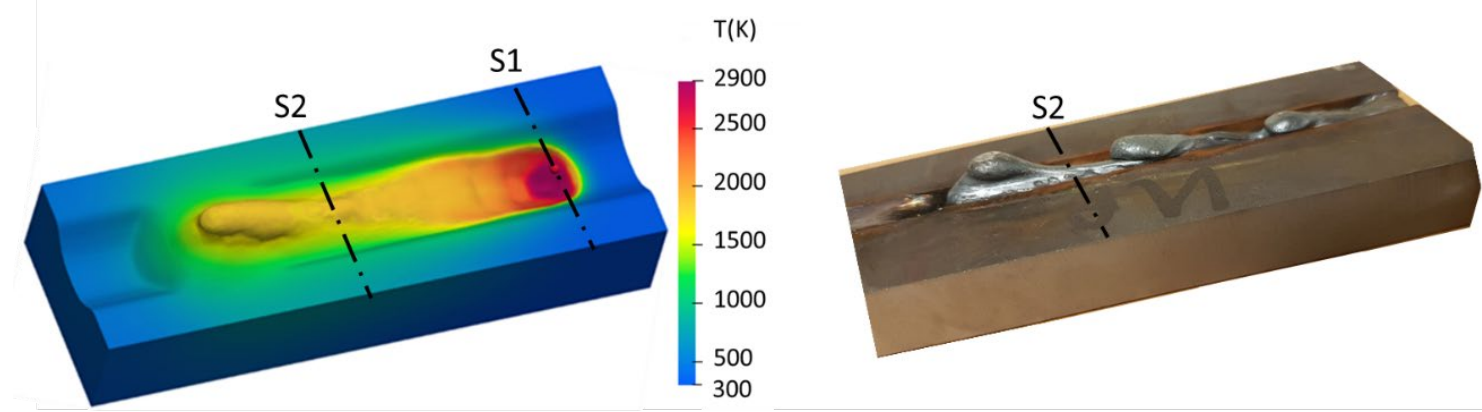

Fig. 3: Bead morphology for the uphill deposit condition. Left: simulation. Right: experiment (different scale).

Figure 4 compares, for each configuration, computational results with experimental macrographs in transverse cross sections. The computed interface between fusion zone (orange/red in Fig. 4) and heat affected zone (yellow in Fig. 4) for the $2^{\text {nd }}$ deposit is plotted at the arc axis location (e.g., section S1 in Fig. 3) when the melt pool has reached quasi-steady dimensions. Superposed on it is the computed bead reinforcement (blue grey in Fig. 4) extracted from the resolidified region (e.g., section S2 in Fig. 3 left). With the flat configuration a) the computed and experimental reinforced bead heights are 0.7 and $0.5 \mathrm{~mm}$ above the original workpiece height, respectively. A small depression that can lead to an undercut defect is observed in the metallograph a). To predict it a finer grid size would be required since its length is much lower than $1 \mathrm{~mm}$. With the downhill configuration $b$ ) the height at the centre of the reinforced bead reaches the level of the original workpiece surface in the experimental metallographs while it is approximately $0.06 \mathrm{~mm}$ lower in the simulations. With the uphill configuration c) and contrary to the other cases, the bead surface is not regular and presents humps as can be seen in Fig. 3. The macrograph image shown in Fig. $4 \mathrm{c}$ ) was obtained from a cross section located at $21 \mathrm{~mm}$ from the arc start for both experiment and simulation. There, the computed and experimental reinforced bead heights are 0.8 and $0.6 \mathrm{~mm}$ above the original workpiece height, respectively. With the side inclined configuration d) the height of the reinforced bead at the centre of the V-groove is at the level of the original workpiece surface in the experimental metallographs. For the numerical simulation it is $0.55 \mathrm{~mm}$ above. Furthermore, the computed bead elevation is slightly lower uphill the groove centre and higher downhill. The undercut defect where the deposited metal fails to fill the groove can be seen on the bead edge in the experimental and computational images. Good qualitative agreement is observed in all the computed conditions. The small 
differences compared to the experimental bead elevation might be due to the simplified arc physics, uncertainty in arc efficiency, or/and to small differences $(\approx 2.5 \%)$ in average value of current and voltage between the cases that were not considered in the simulations.
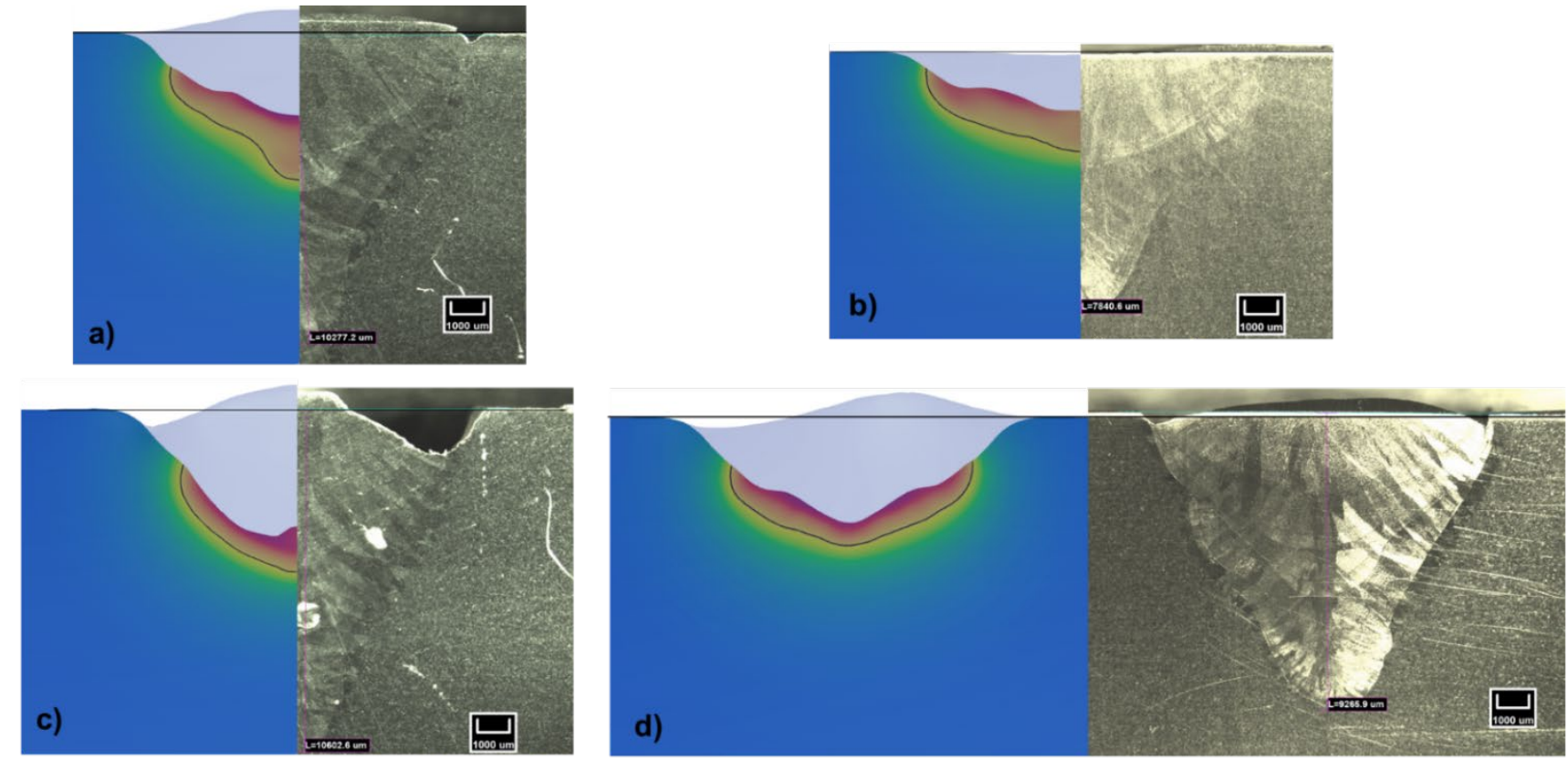

Fig. 4: Computational (left) and experimental (right) bead cross-sections for a) flat, b) uphill, c) downhill, and d) side inclined position.

Figure 5 visualises for each case at time $t=5.0 \mathrm{~s}$ the top view of the fully developed melt pool, its free surface geometry, the temperature field, and the velocity vectors on the free surface. At the rim of the pool crater (corresponding to the red temperature area) the velocity vectors are as expected distributed radially outward due to the thermocapillary force. For the flat deposit a) the maximum velocity outside the pool crater, which also results from the thermocapillary flow, is located in the middle and rear part of the melt where the pool width constricts. A vortex can be seen in the tail end of the melt pool due to the counterflow returning after striking the rear resolidified front. Similar observations can be made for the side inclined position d), but the melt flow is then asymmetric, towards the direction of substrate inclination due to the gravity. The melt pool length is approximately the same in a) and d). Figure 5 shows also that the melt pool is the shortest for the downhill position b) and the longest for the uphill position c). This is due to the gravity force that in the former case supplements the thermocapillary force ahead of the arc to accelerate the flow towards the front part of the melt pool (and decelerate the rear flow). In the latter case it acts on opposite direction, thus accelerates the rear flow (and decelerates the pool front flow) while surface tension is not sufficient to counteract the resultant increased pressure. This can also be seen with the velocity vectors, e.g., the melt flow shows larger velocity vectors towards the forefront in downhill position $b$ ) and towards the pool rear in uphill position c). Also, the maximum velocity is the lowest in b) and the largest in c). Due to the weaker flow for the downhill position b) compared to the other cases, no vortex is observed at the rear part of the melt pool in $b$ ). Besides, these changes in flow velocity compared to the flat case a) induce pressure changes on the free surface that the surface tension does not sustain. Thus, the pool deformation leading to wider pool front in downhill position b), longer pool rear in uphill position c), and asymmetric pool edge in side inclined position d) compared to the flat case a). The temperature distributions within the melt pools are in close ranges, with a maximum in the vicinity of $2920 \mathrm{~K}$ for all cases except the downhill position that is $200 \mathrm{~K}$ colder. The melt pool is also wider for the downhill position $\mathrm{b}$ ) than for the other cases, providing higher degree of cooling rate. The computed melt pool geometries of Fig. 5 are consistent with the top view images of the pool free surface behind the arc acquired during the experiments and shown in Fig 6. These images indeed show the shortest melt pool length for the downhill position $b$ ) and an asymmetric pool with more melt towards the downhill direction (indicated by the white arrow in Fig. 6 d)) of the substrate for the side inclined position. For the uphill position c), the melt pool width is significantly 
narrower, and gaps indicated with arrows can be seen between the melt pool and the groove wall. They contribute to the undercut defects seen in Fig 4.

a)

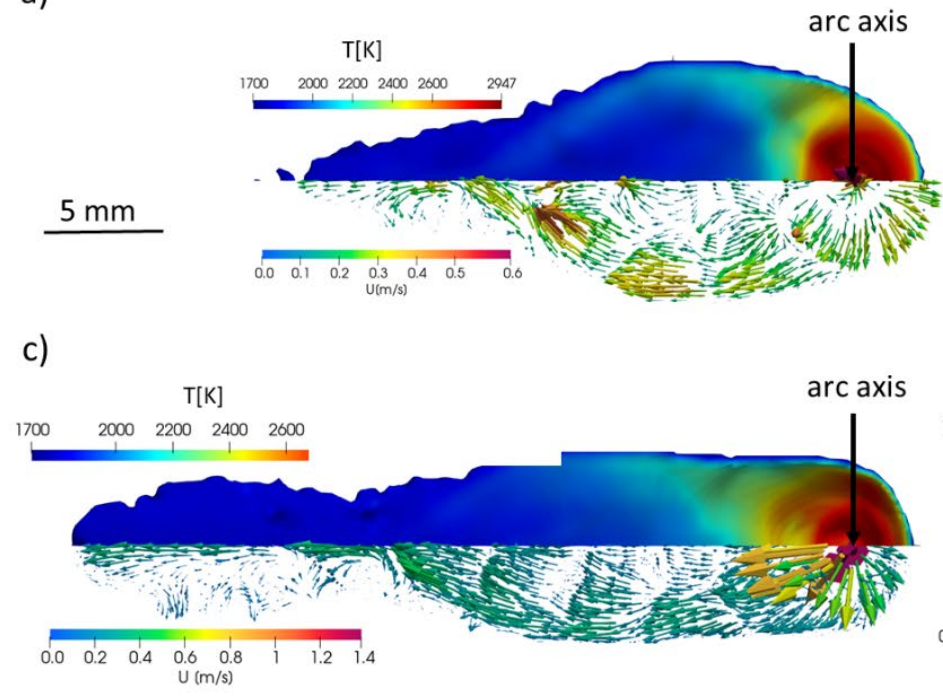

b)

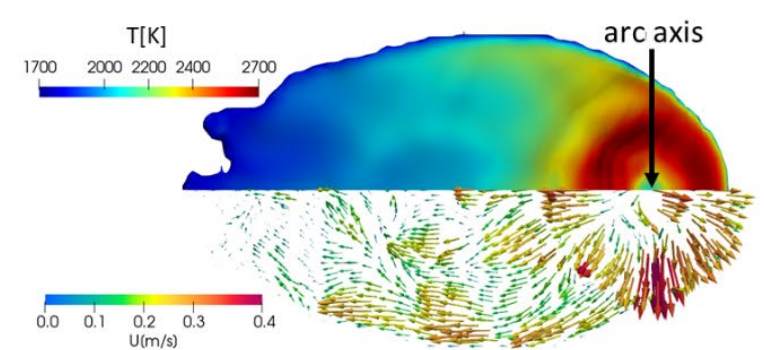

d)

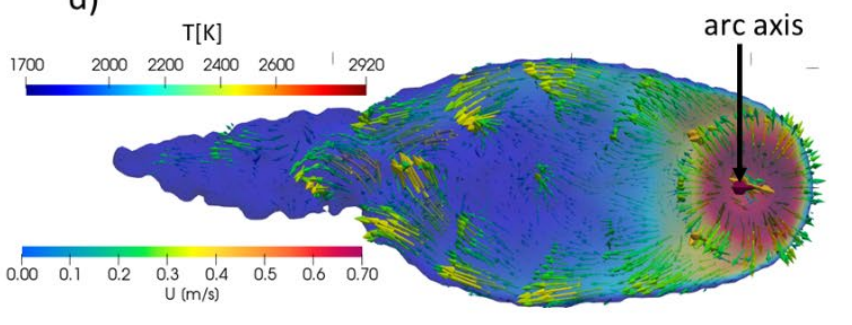

Fig. 5: Temperature and velocity vectors on the computed melt pool free surface; a) flat, b) downhill, c) uphill, d) inclined case.
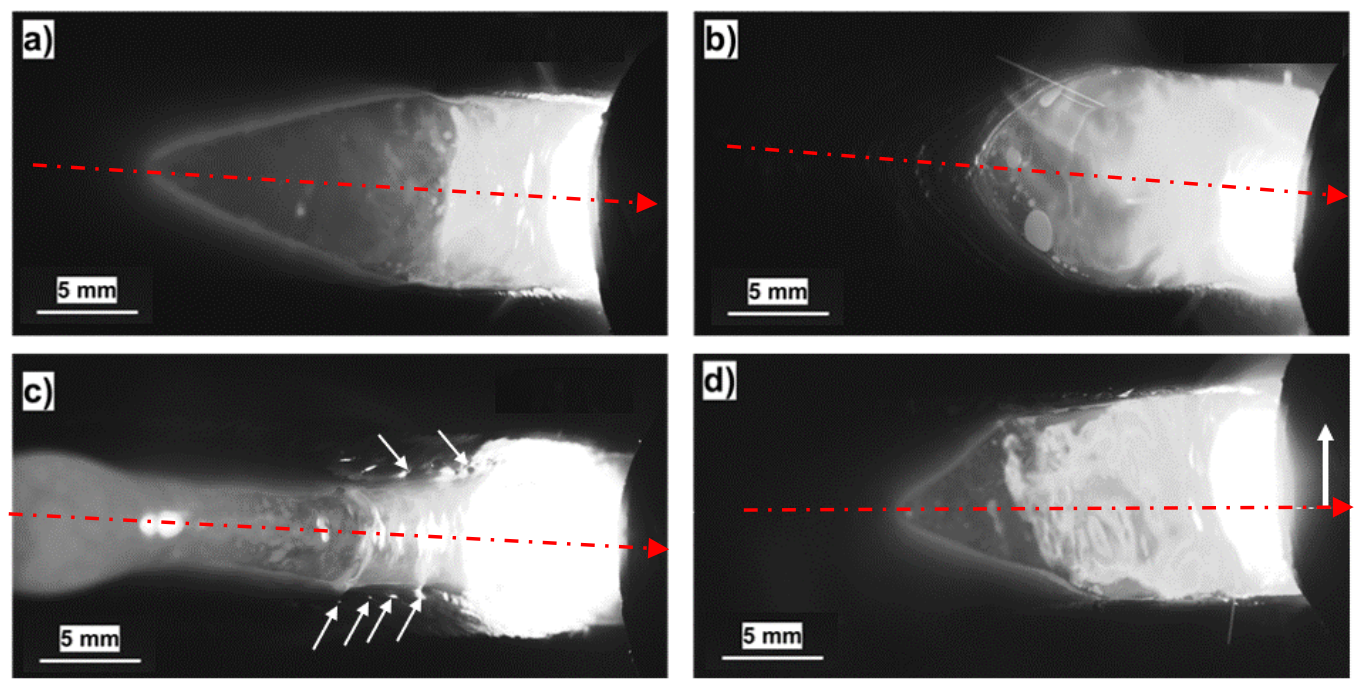

Fig. 6: Melt pool top view captured with a vision camera; a) flat, b) downhill, c) uphill, and d) side inclined (the white arrow points downhill). The red arrows indicate the arc centre travel direction.

Figure 7 shows for each case (at same scale) a longitudinal cross-section passing through the arc axis of the computed melt pool (grey in Fig. 7) and the velocity vectors at time $t=5.0 \mathrm{~s}$ where pool dimensions are quasi-steady. The horizontal red line indicates the upper surface of the first layer deposit. It should be noticed that the velocity vector plotted at the arc axis location orients differently depending on the dynamic formation/restoration of the pool crater with drop impact. It cannot be compared between the instantaneous images a) to d) since although synchronised with the drop detachment frequency the impact is not exactly at the same time as surface oscillations can differ with the configuration. Two regions recirculating in 
opposite directions can be seen in the melt pool in the flat position a). The recirculation flow that is closest to the arc is clockwise, therefore it enhances the heat transfer under the arc and the penetration depth. For the other positions, the clockwise recirculation is absent. For the downhill position b) a significantly shallower melt pool can be observed. The counterclockwise recirculation is then much more extended than in the flat position a) and it flows at a velocity of $\approx 0.3 \mathrm{~m} / \mathrm{s}$, which is about $0.1 \mathrm{~m} / \mathrm{s}$ larger than in the counterclockwise recirculation of a), indicating a stronger returning flow in the shallower melt pool. For the uphill position c) a significant free surface depression can be seen underneath the arc column since a large volume of melted alloy flows downward, towards the pool rear under the action of the thermocapillary force enhanced by the gravitational force. This fluid accumulates in the trailing region, thus the high alloy thickness visible above the red line indicator in Fig. 7 c), resulting in a hump upon solidification. For the side inclined deposit position d) the melt pool shape at the centerplane is relatively shallow compared to the flat deposit position since some of the liquid metal then flows towards the downhill edge (see Fig. 5d).

(a)

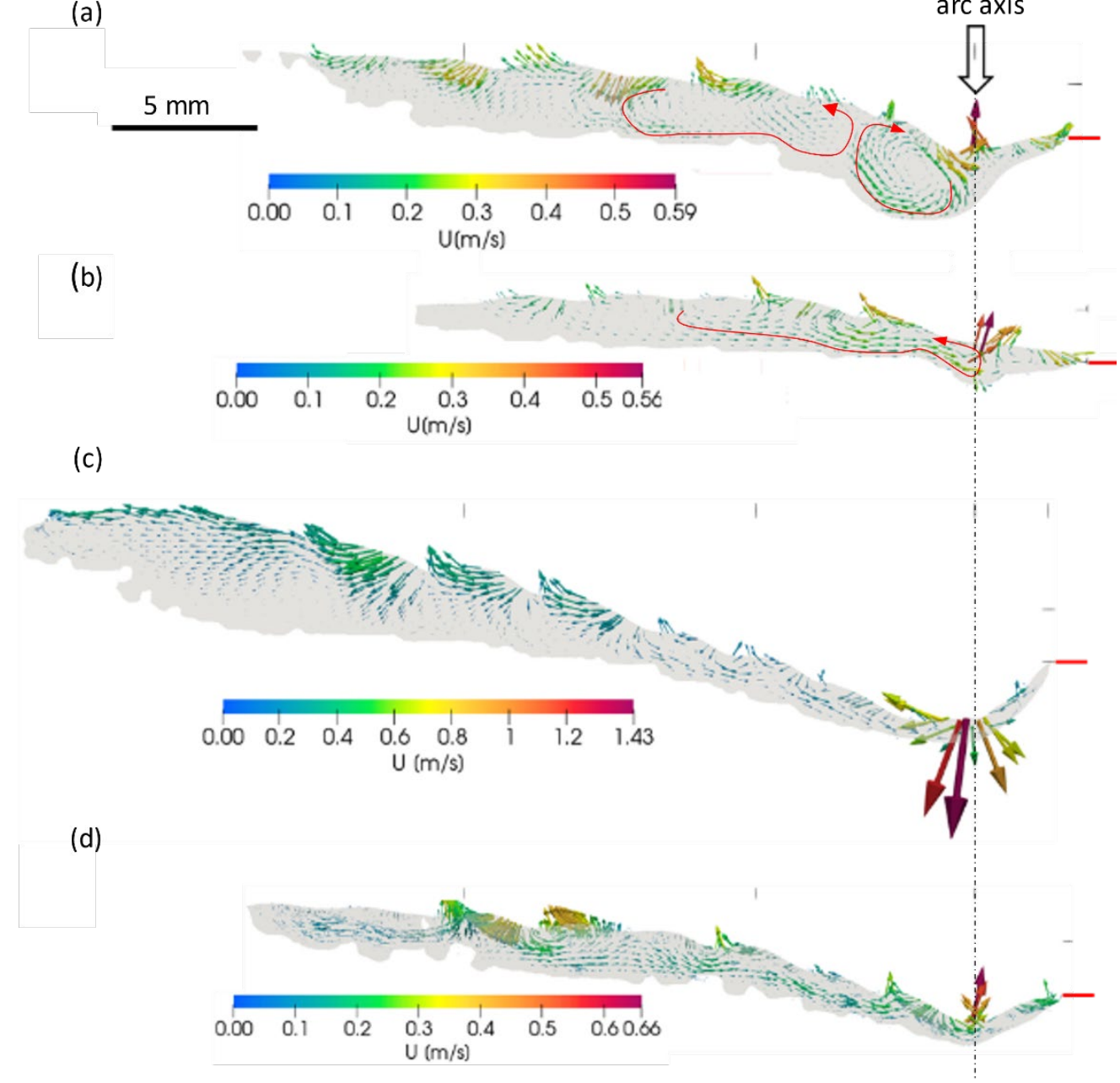

Fig. 7: Melt pool and velocity vectors in longitudinal section through the arc axis for a) flat b) downhill c) uphill d) side position.

\section{Conclusion}

A thermo-fluid model for metal fusion with GMA heat source with tracking of free surface deformation and metal transfer developed in OpenFOAM was applied to study the effect of different substrate orientations on metal layer deposition over a pre-deposited layer in a V-groove. The numerical study was supplemented with experimental observation and the 
results were in good qualitative agreement. However, the undercut defects observed in the experimental metallographs were only partially captured by the numerical simulations. A much finer grid size would be needed for a precise prediction of these small undercut defects. Nevertheless, it was found that deposition of a second layer on a smooth first layer that had been cooled down to room temperature leads,

- for flat welding position, to a liquid alloy recirculation pattern that enhances the penetration depth under the arc, and to smooth and continuous reinforced bead,

- for the $20^{\circ}$ downhill position, to a large and strong returning flow behind the arc axis towards the melt front, which increases both the melt pool width and its cooling, while it decreases its length and the bead height.

- for the $20^{\circ}$ uphill position, to flow acceleration by gravity towards the pool rear, resulting in a narrowing of the pool width, increased pool length, and liquid alloy accumulation at the pool rear resulting in undercuts and bead humps upon solidification.

- for the $20^{\circ}$ side inclined position, the trailing part of the melt pool tends to flow downhill resulting in incomplete fusion on the opposite side of the $\mathrm{V}$ groove and undercut defects.

Therefore, uphill and side inclined multilayer GMA deposition are not recommended with these process conditions. Further investigations are needed to establish the range of inclination angles where acceptable deposition results are maintained.

\section{Acknowledgements}

This research work is supported by grants from the EU project - Horizon 2020: INTEGRADDE, which is gratefully acknowledged. The computations were performed on resources provided by the Swedish National Infrastructure for Computing (SNIC) at NSC which is gratefully acknowledged.

\section{References}

[1] J. H. Park, S. H. Kim, H. S. Moon, and M. H. Kim, "Influence of gravity on molten pool behavior and analysis of microstructure on various welding positions in pulsed gas metal arc welding," Appl. Sci., vol. 9, no. 21, 2019.

[2] Z. Hu, L. Hua, X. Qin, M. Ni, F. Ji, and M. Wu, "Molten pool behaviors and forming appearance of robotic GMAW on complex surface with various welding positions," J. Manuf. Process., vol. 64, no. January, pp. 1359-1376, 2021.

[3] D. W. Cho, S. J. Na, M. H. Cho, and J. S. Lee, "A study on V-groove GMAW for various welding positions," J. Mater. Process. Technol., vol. 213, no. 9, pp. 1640-1652, 2013.

[4] H. Jäger, "Thermophysical properties of liquid Fe64 / Ni36 ( IN," vol. 36, pp. 1004-1008, 1997.

[5] F. Rösler, D. Brüggemann, Shell-and-tube type latent heat thermal energy storage: numerical analysis and comparison with experiments. Heat Mass Transfer, 47 (2011) 1027-1033.

[6] M. Shams, A.Q. Raeini, M.J. Blunt, B. Bijeljic, A numerical model of two- phase flow at the micro-scale using the volume-of-fluid method. Journal of Computational Physics, 357 (2018) 159-182.

[7] J.U. Brackbill, D.B. Kothe, C. Zemach, A continuum method for modeling surface tension. Journal of Computational Physics, 100:2 (1992) 335-354.

[8] O. Ubbink, Numerical prediction of two fluid systems with sharp interfaces. PhD Thesis, Imperial College, University of London, 1997.

[9] N. S. Tsai and T. W. Eagar, "Distribution of the heat and current fluxes in gas tungsten arcs," Metall. Trans. B, vol. 16, no. 4 , pp. 841-846, 1985.

[10] S. Kou and D. K. Sun, "Fluid flow and weld penetration in stationary arc welds," Metall. Trans. A, vol. 16, no. 2, pp. 203-213, 1985.

[11] D. W. Cho, J. H. Park, and H. S. Moon, "A study on molten pool behavior in the one pulse one drop GMAW process using computational fluid dynamics," Int. J. Heat Mass Transf., vol. 139, pp. 848-859, 2019.

[12] J. Cheon, D. V. Kiran, and S. J. Na, "CFD based visualization of the finger shaped evolution in the gas metal arc welding process," Int. J. Heat Mass Transf., vol. 97, pp. 1-14, 2016.

[13] D. W. Cho, S. H. Lee, and S. J. Na, "Characterization of welding arc and weld pool formation in vacuum gas hollow tungsten arc welding," J. Mater. Process. Technol., vol. 213, no. 2, pp. 143-152, 2013. 